\title{
Gas, Dust and Star Formation in Nearby Galaxies as Seen with the JCMT
}

\author{
José Ramón Sánchez-Gallego ${ }^{1,2}$ \\ ${ }^{1}$ Instituto de Astrofísica de Canarias, \\ E-38205, La Laguna, Tenerife, Spain \\ ${ }^{2}$ Departamento de Astrofísica, Universidad de La Laguna, \\ E-38200 La Laguna, Tenerife, Spain \\ email: jrsg@iac.es
}

\begin{abstract}
We combine ${ }^{12} \mathrm{CO} J=3-2$ for 155 nearby galaxies, obtained as part of the James Clerk Maxwell Telescope (JCMT) Nearby Galaxies Legacy Survey (NGLS), with star formation rates derived from $\mathrm{H} \alpha$ luminosities to probe the relation between warm, dense gas and star formation. These data are compared with ancillary data from the literature, including infrared (IR) and $1.4 \mathrm{GHz}$ radio continuum luminosities and $\mathrm{HI}$ masses. We find a good correlation between CO $J=3-2$ luminosities and $\mathrm{H} \alpha$-derived star formation rates (SFRs), which is in agreement with previous studies. Several groups of galaxies show a peculiar behaviour in the $\operatorname{SFR}(\mathrm{H} \alpha)-L_{\mathrm{CO}} J_{=3-2}$ plot, which disappears when using IR data. We analyse these regions and propose several hypotheses to explain this behaviour, including group interactions and very recent $(\lesssim 20 \mathrm{Myr})$ bursts of star formation only traced by $\mathrm{H} \alpha$ emission. While atomic hydrogen is hardly related with SFR, molecular and total gas $\left(\mathrm{H} \mathrm{I}+\mathrm{H}_{2}\right)$ show similar correlations with $\mathrm{H} \alpha$ luminosities. No correlation is found between CO $J=3-2$ luminosity and metallicity, although galaxies with low metallicity are more likely to not be detected. We discuss that $\mathrm{CO} J=3-2$ does not seem to be a significantly better tracer of the star-forming gas, but its independence from metallicity may be used to constrain the conversion factor between $\mathrm{CO}$ and $\mathrm{H}_{2}$.
\end{abstract}

Keywords. galaxies: ISM — ISM: molecules — stars: formation

\section{Introduction}

Star formation is the most important factor controlling the evolution of galaxies, with the possible exception of galaxy-galaxy interaction, and has become one of the most researched and discussed fields in today's astronomy. The details of the interplay between the gas content of galaxies and the processes triggering star formation are complex, mainly due to the difficulties involved in measuring star formation rates and molecular hydrogen, even in nearby galaxies. While challenging, the field is key in understanding the formation and evolution of galaxies, both nearby and at cosmological distances, and new facilities such as Herschel and ALMA promise an exciting future for the researchers working on these subjects.

Important efforts have been made in the last decades both to understand the physics behind the birth of stars and to measure the pace of star formation. The subject has been extensively researched both in the local Universe (e.g., Kennicutt \& Kent 1983; Gallego et al. 1995; Alonso et al. 1999; James et al. 2004; Kennicutt et al. 2008) and in the more distant Universe (e.g., Madau et al. 1996; Hopkins et al. 2000; Lanzetta et al. 2002; Nagamine et al. 2006). However, the matter is complex and many fundamental questions in Galactic and extragalactic research remain. 


\section{The Nearby Galaxies Legacy Survey}

The NGLS is part of a larger series of ongoing legacy surveys being carried out at the James Clerk Maxwell Telescope (JCMT) on Mauna Kea, Hawai'i. The survey entails two major phases: a first set of observations has mapped the whole sample of galaxies in the ${ }^{12} \mathrm{CO} J=3-2$ line to trace the warm, dense molecular gas, and the second phase will obtain continuum imaging at 450 and $850 \mu \mathrm{m}$ using SCUBA-2 (Dempsey et al. 2012). The scientific goals pursued include the analysis of the molecular gas and dust, as well as the gas-to-dust ratio over wide morphological and environmental ranges.

To avoid a star formation-driven selection while ensuring that the galaxies chosen have a rich interstellar medium, $\mathrm{H}$ I fluxes were used to compile the galaxies for the sample. We used HyperLEDA (Paturel et al. 2003) to select all the non-Virgo galaxies with Virgo-corrected velocities lower than $1875 \mathrm{~km} \mathrm{~s}^{-1}$ and distances between 2 and $25 \mathrm{Mpc}$. From this initial selection we took the 47 galaxies matching the sample of the Spitzer Infrared Nearby Galaxies Survey (SINGS; Kennicutt et al. 2003). Another 36 galaxies were randomly selected from the Virgo Cluster. The remaining galaxies, to a total of 155 , were randomly chosen to include a statistically representative number of galaxies from early- to late-type and irregulars. The morphological breakdown of our sample is shown in Figure 1 of Sánchez-Gallego et al. (2012). Further details about the selection of the sample, data acquisition and reduction can be found in Wilson et al. (2009, 2012) and Warren et al. (2010).

We have also obtained new $\mathrm{H} \alpha$ continuum-subtracted imaging for 72 of the galaxies in the NGLS sample (Sánchez-Gallego et al. 2012). For the remaining 83 galaxies, we compiled $\mathrm{H} \alpha$ imaging from the literature (James et al. 2004; Kennicutt 2003; Knapen et al. 2004; Kennicutt et al. 2008; and the GOLDMine database $)$ ).

\section{Results and discussion}

Our analysis of the $\mathrm{H} \alpha$-derived SFRs and $\mathrm{CO} J=3-2$ luminosities shows (Figure 1) a general good correlation between both magnitudes. We find a correlation factor $r=0.74$, which is in good agreement with other studies using lower transitions of the CO molecules (see, for example, Corbelli et al. 2012). Several groups of galaxies (marked in Figure 1 as regions $\mathrm{A}$ to $\mathrm{D}$ ) show an unexpected behaviour, lying outside the global trend. Most interestingly, this behaviour seems to disappear when we use infrared fluxes instead of $\mathrm{H} \alpha$ SFRs. We have studied in detail these regions and found no important differences between the galaxies that constitute them. These galaxies are mostly intermediate-spirals (which is to be expected, as most of the early-type galaxies in our sample have only been detected as upper limits) with H I masses and non-thermal radio continuum fluxes that are well within the expected range for galaxies with their CO $J=3-2$ luminosities. Almost every galaxy that shows a peculiar behaviour has close companions or belongs to a group, and we suggest that group membership may indeed be an important factor that can trigger or inhibit the star formation in these galaxies.

Now, unlike infrared, $\mathrm{H} \alpha$ gives us a measure of the young star formation rate, as the line is excited by luminous, short-lived $\mathrm{OB}$ conglomerates. We have also shown that galaxies that have higher SFRs than expected for their CO luminosities display higherthan-average EWs. The inverse is true for galaxies with low SFRs for their measured $L_{\mathrm{CO} \quad J=3-2}$. Equivalent width $(\mathrm{EW})$ is a good indicator of how significant the star formation is with respect to the total luminosity of the galaxy (i.e., its mass). In this case, the combination of high $\mathrm{H} \alpha$-derived SFR and high $\mathrm{EW}(\mathrm{H} \alpha)$ (or low $\mathrm{SFR}$ and low $\mathrm{EW}$ for

$\dagger$ http://goldmine.mib.infn.it/ 
regions $\mathrm{C}$ and $\mathrm{D}$ ) seems to indicate that the odd behaviour of these galaxies is only due to bursts (or the lack thereof) of young massive stars occurring during the last $\sim 20 \mathrm{Myr}$. This scenario explains the fact that these galaxies fit well when using other tracers of star formation such as infrared or non-thermal radio continuum emission, which are more sensitive to slightly older star formation.

One of the main goals of the NGLS is to probe the warm, dense gas traced by the CO $J=3-2$ line that, previously to this study, had not been widely observed in galaxies. We have found that the correlation between $\mathrm{CO} J=3-2$ luminosities and different tracers of star formation is comparable to that found using the CO $J=1-0$ line, but not better. This indicates either that the warm and cold molecular hydrogen present in a galaxy are fairly proportional or that the star formation is more connected otothe cold gas than what recent studies have suggested (e.g., Iono et al. 2009). In any case, further analysis remain to be done on how warm gas correlates with star formation, especially in the range of resolved GMCs.

Finally, as shown in Figure 2, we find little correlation between $\mathrm{CO} J=3-2$ and metallicity. This fact is interesting, as recent studies (e.g., Schruba et al. 2012, using the CO $J=2-1$ line) have shown a clear dependency between the two quantities in a sample that partly intersects with our own. We find, however, that galaxies not detected in CO $J=3-2$ (or detected only as upper limits) are more likely to have low metallicities. As metal-richness has proven to be a key dependency for the $X_{\mathrm{CO}-\mathrm{to}_{-}-\mathrm{H}_{2}}$ factor when using lower transitions of the $\mathrm{CO}$ line, the fact that $\mathrm{CO} J=3-2$ may be independent (or at least, less dependent) with metallicity shows great promise for the use of this line to trace

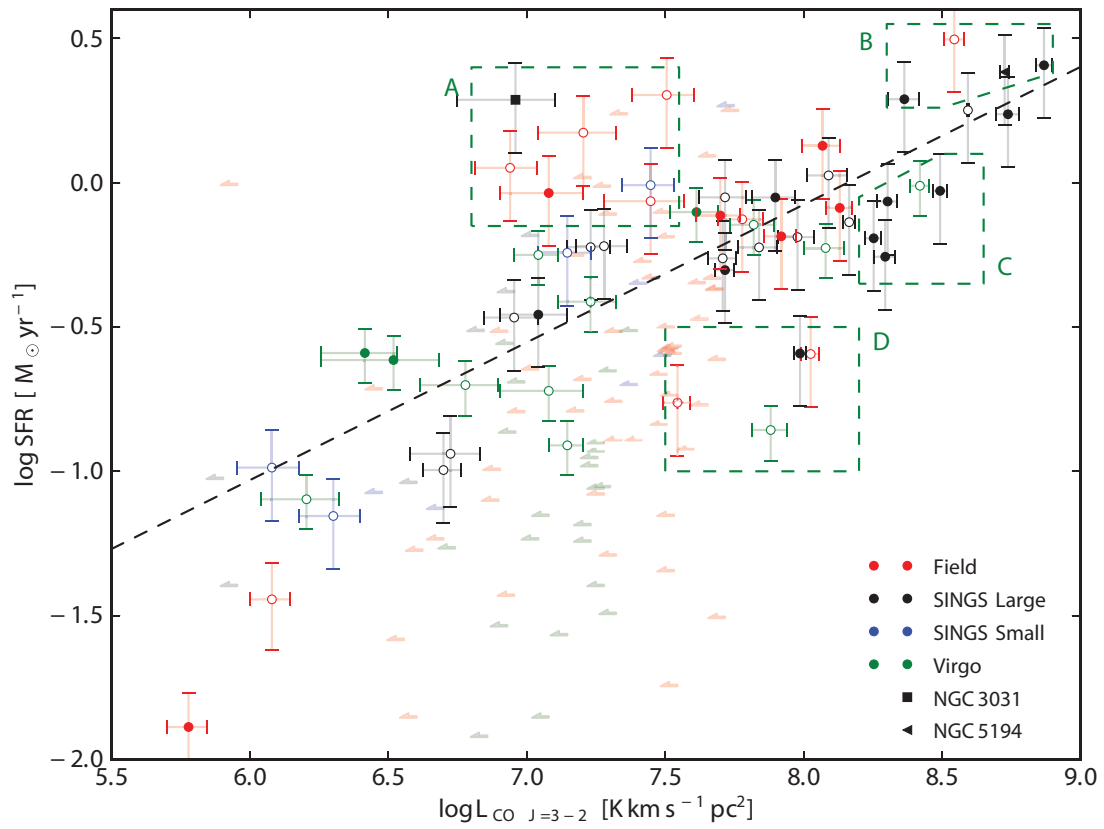

Figure 1. CO $J=3-2$ luminosities versus SFRs from H $\alpha$ measurements. The different subsamples have been plotted in different colours. Different symbols are used for NGC 3031 (M 81) and NGC 5194 (M 51) as a reference. Filled symbols indicate that the galaxy hosts nuclear AGN activity. Upper limit detections have been represented using arrows. The dashed black line is the least squares fit for the galaxies that have been detected. Dashed green rectangles mark the bounds of the regions with peculiar beaviour that are referred in the text. 


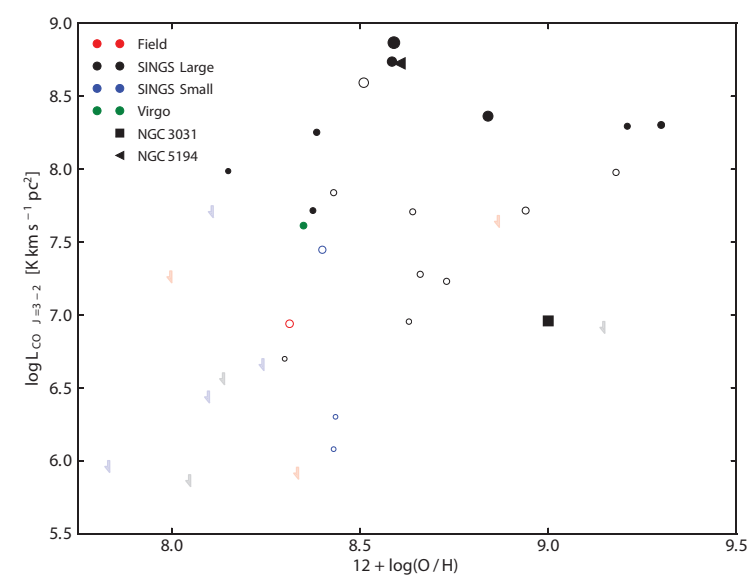

Figure 2. Metallicty, expressed as $12+\log (\mathrm{O} / \mathrm{H})$, versus the logarithm of the CO $J=3-2$ luminosity. The meaning of the symbols is as in Figure 1. The size of each symbol is a function of the SFR of the galaxy.

$\mathrm{H}_{2}$ mass. Furthermore, molecular gas determinations made using the $\mathrm{CO} J=3-2$ lines can be as a diagnostic tool to calibrate the $X$-factor over a wider range of metallicities.

\section{References}

Alonso, O., García-Dabó, C. E., Zamorano, J., Gallego, J., \& Rego, M. 1999, ApJS, 122, 415

Bendo, G. J., Wilson, C. D., Warren, B. E., et al. 2010, MNRAS, 402, 1409

Corbelli, E., Bianchi, S., Cortese, L., et al. 2012, A\&SA, 542, A32

Dempsey, J. T., Holland, W. S., Chrysostomou, A., et al. 2012, ArXiv e-prints (1208.4622)

Gallego, J., Zamorano, J., Aragon-Salamanca, A., \& Rego, M. 1995, ApJL, 455, L1

Hopkins, A. M., Connolly, A. J., \& Szalay, A. S. 2000, AJ, 120, 2843

Iono, D., Wilson, C. D., Yun, M. S., et al. 2009, ApJ, 695, 1537

Irwin, J. A., Wilson, C. D., Wiegert, T., et al. 2011, MNRAS, 410, 1423

James, P. A., Shane, N. S., Beckman, J. E., et al. 2004, A\&SA, 414, 23

Kennicutt, Jr., R. C. \& Kent, S. M. 1983, AJ, 88, 1094

Kennicutt, R. C., Armus, L., Bendo, G., et al. 2003, Publ. Astron. Soc. Pac., 115, 928

Kennicutt, Jr., R. C., Lee, J. C., Funes, José G., S. J., Sakai, S., \& Akiyama, S. 2008, ApJS, 178,247

Knapen, J. H., Stedman, S., Bramich, D. M., Folkes, S. L., \& Bradley, T. R. 2004, A\&AA, 426, 1135

Lanzetta, K. M., Yahata, N., Pascarelle, S., Chen, H.-W., \& Fernández-Soto, A. 2002, ApJ, 570, 492

Madau, P., Ferguson, H. C., Dickinson, M. E., et al. 1996, MNRAS, 283, 1388

Nagamine, K., Ostriker, J. P., Fukugita, M., \& Cen, R. 2006, ApJ, 653, 881

Paturel, G., Petit, C., Prugniel, P., et al. 2003, A\&GA, 412, 45

Sánchez-Gallego, J. R., Knapen, J. H., Heiner, J. S., et al. 2011, A\&\&A, 527, A16

Sánchez-Gallego, J. R., Knapen, J. H.,Wilson, C. D., et al. 2012, MNRAS, 422, 3208

Scoville, N. Z., Polletta, M., Ewald, S., et al. 2001, AJ, 122, 3017

Schruba, A., Leroy, A. K., Walter, F., et al. 2012, AJ, 143, 138

Warren, B. E., Wilson, C. D., Israel, F. P., et al. 2010, ApJ, 714, 571

Wilson, C. D., Warren, B. E., Israel, F. P., et al. 2009, ApJ, 693, 1736

Wilson, C. D., Warren, B. E., Israel, F. P., et al. 2012, MNRAS, 424, 3050 